\title{
The effect of audible sound on protein crystallization
}

Chen-Yan Zhang ${ }^{\mathrm{a}, \dagger}$, Yan Wang ${ }^{\mathrm{a}, \uparrow}$, Robin Schubert ${ }^{\mathrm{b}}$, Yue Liu ${ }^{\mathrm{a}}$, Meng-Yin Wang ${ }^{\mathrm{a}}$, Da

Chen, Yun-Zhu Guo ${ }^{\mathrm{a}}$, Chen Dong ${ }^{\mathrm{a}}$, Hui-Meng Lu ${ }^{\mathrm{a}}$, Yong-Ming Liu ${ }^{\mathrm{a}}$, Zi-Qing Wu${ }^{\mathrm{a}}$, Christian Betzel $^{\mathrm{b}, *}$, and Da-Chuan Yin ${ }^{\mathrm{a}, *}$

${ }^{a}$ Institute for Special Environmental Biophysics, Key Laboratory for Space Bioscience and Biotechnology, School of Life Sciences, Northwestern Polytechnical University, Xi'an 710072, Shaanxi, PR China

${ }^{\mathrm{b}}$ University of Hamburg, Laboratory for Structural Biology of Infection \& Inflammation, Institute for Biochemistry and Molecular Biology, Notkestrasse 85, 22607 Hamburg, Germany

${ }^{\dagger}$ C.Y.Z. and Y.W. contributed equally to this work. 


\section{Supporting Information}

\section{SI Supplementary Figures}

\begin{tabular}{|l|l|l|}
\hline SI 1 & $\begin{array}{l}\text { Fig. S1. Crystal number with and without audible sound. Total } \\
\text { number of crystals with and without audible sound }\end{array}$ & P3 \\
\hline SI 2 & $\begin{array}{l}\text { Fig. S2. Examples of crystal morphology with (a-d) and without (a'- } \\
\text { d') } 1000 \mathrm{~Hz} \text { audible sound. }\end{array}$ & P4 \\
\hline
\end{tabular}

\section{SI Supplementary Tables}

\begin{tabular}{|l|l|l|}
\hline SI 3 & Table S1. Proteins and their buffers used in the investigation & P5 \\
\hline SI 4 & $\begin{array}{l}\text { Table S2. Number of crystallization hits with and without sound } \\
\text { irradiation in verification study in University of Hamburg }\end{array}$ & P6 \\
\hline SI Supplementary Video & $\begin{array}{l}\text { Video 1. DLS monitoring of the particle size evolution against time } \\
\text { with and without sound irradiation. }\end{array}$ & P7 \\
\hline SI &
\end{tabular}




\section{SI Supplementary Figures}

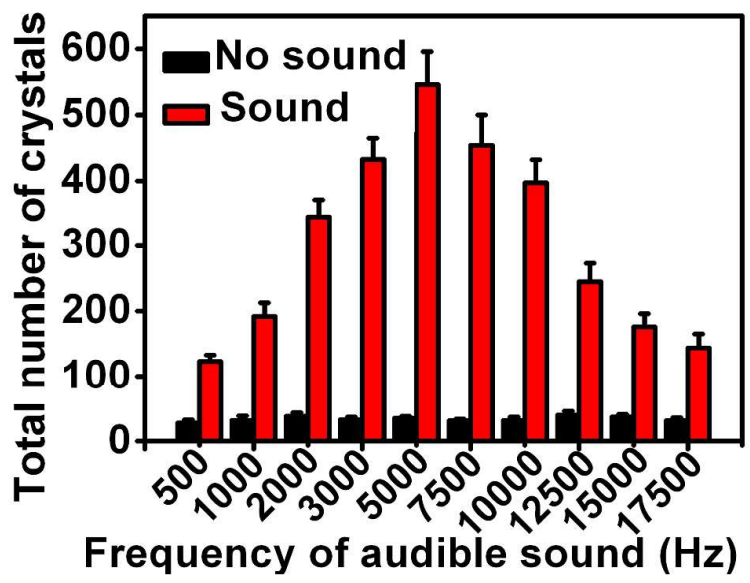

Fig. S1. Crystal number with and without audible sound. Total number of crystals with and without audible sound. Crystallization conditions after mixing: $20 \mathrm{mg} / \mathrm{ml}$ lysozyme, $40 \mathrm{mg} / \mathrm{ml} \mathrm{NaCl}$ (Error bar: standard deviation, $\mathrm{n}=30$ ). It demonstrated that nucleation is correlative with frequency of audible sound. 


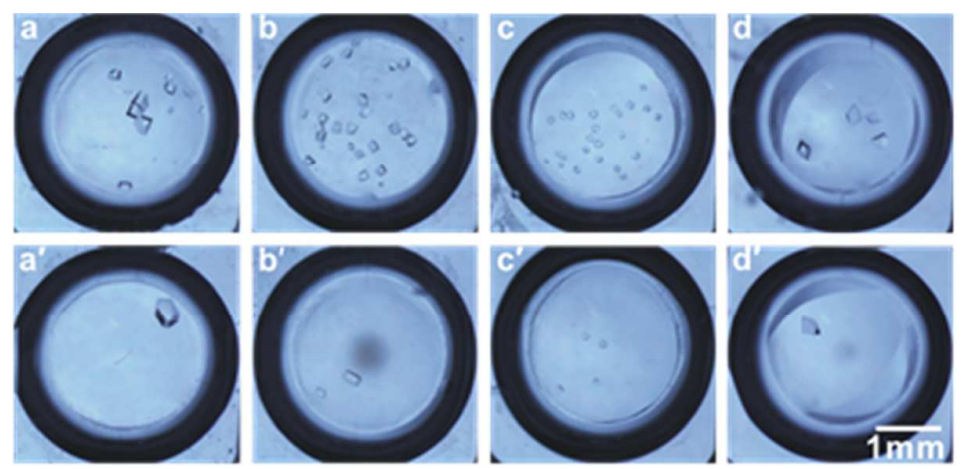

Fig. S2. Examples of crystal morphology with (a-d) and without $\left(a^{\prime}-d^{\prime}\right) 1000 \mathrm{~Hz}$ audible sound. a and $\mathrm{a}^{\prime}$ : lysozyme; b and $\mathrm{b}^{\prime}$ : concanavalin $\mathrm{A} ; \mathrm{c}$ and $\mathrm{c}^{\prime}$ : glucose isomerase; $d$ and $\mathrm{d}^{\prime}$ : proteinase $\mathrm{K}$. The crystallization technique was sitting-drop method. 


\section{SI Supplementary Tables}

Table S1. Proteins and their buffers used in the investigation

\begin{tabular}{|c|c|c|c|c|}
\hline Proteins & Abbreviate & Cata No. & Suppliers & Buffers \\
\hline \multicolumn{5}{|c|}{ Followings are proteins used at Northwestern Polytechnical University for sound effect study } \\
\hline Lysozyme & Lys. & E05801 & Seikagaku & $100 \mathrm{mM}$ Sodium acetate, $\mathrm{pH} 4.6$ \\
\hline Catalase & Cat. & $\mathrm{C} 40$ & Sigma-Aldrich & 25 mM HEPES sodium, $\mathrm{pH} 7.0$ \\
\hline Subtilisin AVII & Sub. & P5380 & Sigma-Aldrich & 25 mM HEPES sodium, $\mathrm{pH} 7.0$ \\
\hline Thaumatin & Thau. & T7638 & Sigma-Aldrich & 25 mM HEPES sodium, $\mathrm{pH} 7.0$ \\
\hline Insulin & Ins. & $\mathrm{Z} 5500$ & Sigma-Aldrich & $100 \mathrm{mM}$ Ammonia \\
\hline Concanavalin A VI & Con. & L7647 & Sigma-Aldrich & 25 mM HEPES sodium, $\mathrm{pH} 7.0$ \\
\hline Ribonuclease A I & Rib. I & $\mathrm{R} 4875$ & Sigma-Aldrich & 25 mM HEPES sodium, $\mathrm{pH} 7.0$ \\
\hline Ribonuclease A III & Rib. III & R5125 & Sigma-Aldrich & 25 mM HEPES sodium, $\mathrm{pH} 7.0$ \\
\hline Ribonuclease A XII & Rib. XII & $\mathrm{R} 5500$ & Sigma-Aldrich & 25 mM HEPES sodium, $\mathrm{pH} 7.0$ \\
\hline$\alpha$-chymotrypsinogen AII & Chy. & C4879 & Sigma-Aldrich & 25 mM HEPES sodium, $\mathrm{pH} 7.0$ \\
\hline Proteinase $\mathrm{K}^{\mathrm{a}}$ & Prok. & P6556 & Sigma-Aldrich & 25 mM HEPES sodium, $\mathrm{pH} 7.0$ \\
\hline Cellulase & Cel. & $\mathrm{C} 0615$ & Sigma-Aldrich & 25 mM HEPES sodium, $\mathrm{pH} 7.0$ \\
\hline Papain & Pap. & P3125 & Sigma-Aldrich & 25 mM HEPES sodium, $\mathrm{pH} 7.0$ \\
\hline Lactalbumin & Lac. & K7015 & Sigma-Aldrich & 25 mM HEPES sodium, $\mathrm{pH} 7.0$ \\
\hline Glucose Isomerase & Glu. & HR7-100 & Hampton Research & 25 mM HEPES sodium, $\mathrm{pH} 7.0$ \\
\hline \multicolumn{5}{|c|}{ Followings are proteins used at University of Hamburg for sound effect verification study } \\
\hline Proteinase K & Prok. & 105281 & Merck Millipore & 25 mM HEPES sodium, $\mathrm{pH} 7.0$ \\
\hline Thaumatin & Thau. & T7638 & Sigma-Aldrich & 25 mM HEPES sodium, pH7.0 \\
\hline Lysozyme & Lys. & HR7-110 & Hampton Research & $50 \mathrm{mM}$ Sodium acetate, $\mathrm{pH} 4.6$ \\
\hline Lipase B & Lipb. & HR7-099 & Hampton Research & 25 mM HEPES sodium, $\mathrm{pH} 7.0$ \\
\hline Ribonuclease A I & Rib. I & $\mathrm{R} 4875$ & Sigma-Aldrich & 25 mM HEPES sodium, $\mathrm{pH} 7.0$ \\
\hline Carbonic Anhydrase & Carb. & C3934 & Sigma-Aldrich & 25 mM HEPES sodium, $\mathrm{pH} 7.0$ \\
\hline$\alpha$-Glucosidase & $\alpha$-Glu. & G0660 & Sigma-Aldrich & 25 mM HEPES sodium, $\mathrm{pH} 7.0$ \\
\hline Superoxide Dismutase & Sup. & $\mathrm{S} 2515$ & Sigma-Aldrich & 25 mM HEPES sodium, pH7.0 \\
\hline
\end{tabular}

a: The lot number of proteinase $\mathrm{K}$ utilized at $1000 \mathrm{~Hz}$ and $5000 \mathrm{~Hz}$ was $119 \mathrm{~K} 8602$ and $039 \mathrm{~K} 8618$, respectively. 
Table S2. Number of crystallization hits with and without sound irradiation in verification study in University of Hamburg

\begin{tabular}{|c|c|c|c|c|c|}
\hline \multirow[t]{2}{*}{ Proteins } & \multirow[t]{2}{*}{ Screen kits } & \multicolumn{2}{|c|}{ No sound } & \multicolumn{2}{|c|}{$5000 \mathrm{~Hz}$} \\
\hline & & Rep. 1 & Rep.2 & Rep.1 & Rep.2 \\
\hline \multirow[t]{2}{*}{ Proteinase K $(20 \mathrm{mg} / \mathrm{ml})$} & Classics & 5 & 3 & 10 & 10 \\
\hline & Index HR & 0 & 0 & 3 & 1 \\
\hline \multirow[t]{2}{*}{ Thaumatin $(20 \mathrm{mg} / \mathrm{ml})$} & Classics & 1 & 1 & 3 & 1 \\
\hline & Index HR & 1 & 1 & 3 & 2 \\
\hline \multirow{3}{*}{ Lysozyme (20 mg/ml) } & Classics & 12 & 9 & 12 & 11 \\
\hline & JCSG & 8 & 8 & 15 & 18 \\
\hline & Index HR & 14 & 13 & 16 & 14 \\
\hline Lipase B (20 mg/ml) & Index HR & 12 & 12 & 10 & 10 \\
\hline Ribonuclease A1 (24 mg/ml) & Index HR & 0 & 0 & 1 & 1 \\
\hline Carbonic Anhydrase $(14 \mathrm{mg} / \mathrm{ml})$ & Index HR & 1 & -- & 1 & -- \\
\hline$\alpha$-Glucosidase $(9 \mathrm{mg} / \mathrm{ml})$ & Index HR & 0 & -- & 3 & -- \\
\hline Superoxide Dismutase $(4.5 \mathrm{mg} / \mathrm{ml})$ & Index HR & 8 & -- & 9 & -- \\
\hline
\end{tabular}

Note: in total 21 comparisons were conducted. Among these comparisons, 16 showed positive effect under sound irradiation, and 3 showed the same effect with and without sound irradiation. Only 2 showed reversed results. We sampled all comparisons and applied one-sample $t$ - test to the results. This result showed that sound irradiation at $5000 \mathrm{~Hz}$ revealed a significant effect on the number of protein crystallization hits $(\mathrm{P}=0.0264<0.05)$. 


\section{SI Supplementary Video}

Video 1. DLS monitoring of the particle size evolution against time with and without sound irradiation.

This movie contains in-situ monitoring of the cluster size evolution in the crystallization solution against time with and without sound irradiation. It shows that the cluster size is more homogeneous when the sound irradiation is applied than that without sound irradiation. The result verified that sound irradiation indeed affects the solution status which may cause difference in protein crystallization. 